\title{
THE VOLUME OF A HYPERBOLIC 3-MANIFOLD WITH BETTI NUMBER 2
}

\author{
MARC CULLER AND PETER B. SHALEN
}

(Communicated by James West)

\begin{abstract}
If $M$ is a closed orientable hyperbolic 3-manifold with first Betti number 2 then the volume of $M$ exceeds 0.34 .
\end{abstract}

\section{INTRODUCTION}

It was shown in [2j that if $M$ is a closed orientable hyperbolic 3-manifold for which the first Betti number $\beta_{1}(M)$ is at least 3, then the volume of $M$ is at least 0.92 . In this note we obtain a volume estimate of the same order of magnitude under the weaker hypothesis $\beta_{1}(M) \geq 2$ :

Theorem. If $M$ is a closed orientable hyperbolic 3-manifold with $\beta_{1}(M) \geq 2$ then the volume of $M$ exceeds 0.34 .

In a forthcoming paper [4] we will show that if one excludes certain special manifolds, such as fiber bundles over $S^{1}$, then the same estimate holds for hyperbolic manifolds with Betti number 1 .

Our volume estimates can be placed in context by comparing them with volume estimates for general hyperbolic manifolds, as well as with volumes of known examples. The largest known lower bound for the volume of an arbitrary closed orientable hyperbolic 3-manifold is 0.00115 . This is a result of Gehring and Martin [5], who improved an earlier estimate of 0.00082 due to Meyerhoff. An excellent source of examples of hyperbolic manifolds of small volume is the census conducted by Weeks of hyperbolic 3-manifolds which can be constructed from at most seven ideal tetrahedra. Among the closed manifolds listed in the census the smallest example is orientable with $\beta_{1}=0$ and has a volume of approximately 0.94 . There are at most eight distinct manifolds in the census with nonzero $\beta_{1}$, and they are all orientable with $\beta_{1}=1$. The smallest volume among these examples is approximately 2.78 .

The volume estimate when $\beta_{1} \geq 3$ is a corollary of the main theorem of [2], which implies that if $M$ is a closed orientable hyperbolic 3-manifold and every 2-generator subgroup of $\pi_{1}(M)$ is topologically tame and of infinite index, then $M$ contains an open set which is isometric (as a Riemannian manifold) to a

Received by the editors July 28, 1992.

1991 Mathematics Subject Classification. Primary 57M50; Secondary 57N10.

Both authors are partially supported by the National Science Foundation. 
ball of radius $(\log 3) / 2$ in hyperbolic space. The condition $\beta_{1}(M) \geq 3$ implies that all 2-generator subgroups of $\pi_{1}(M)$ are topologically tame and of infinite index. Density estimates for hyperbolic sphere packings imply that a hyperbolic manifold which contains a hyperbolic ball of radius $(\log 3) / 2$ must have volume at least 0.92 . The estimate in this note is also based on the " $\log 3$ Theorem" of [2] but combines it with a trade-off argument similar to that of [8] (see also [6]), where the volume estimate is obtained by proving that either $M$ contains a hyperbolic ball of a certain radius or else it contains a tubular neighborhood of a closed geodesic for which the geometry is sufficiently well prescribed to allow estimation of its volume.

To obtain a quantitative estimate of the volume, we need to carry out a detailed analysis of the geometry of "displacement cylinders" for hyperbolic isometries. Suppose that $x$ is a loxodromic isometry of hyperbolic 3-space which has translation length $\lambda<\log 3$ along its axis. Consider the " $(\log 3)$ cylinder" consisting of the points of $\mathbf{H}^{3}$ which are moved a distance less than $\log 3$ by $x$. This is a circular cylinder centered on the axis of $x$; i.e., it consists of all points within some fixed distance $R$ of the axis of $x$. However, in contrast to the two-dimensional situation, the radius $R$ is not a function of the translation length of $x$. The action of $x$ on $\mathbf{H}^{3}$ involves both a translation through a distance $\lambda$ along the axis and a rotation around the axis through a "twist angle" $\theta$. (The twist $\theta$ can be defined as the dihedral angle between $P$ and $x(P)$ where $P$ is any plane containing the axis of $x$.) The radius $R$ depends on both $\lambda$ and $\theta$. Moreover, it is possible for the $(\log 3)$-cylinder associated with a power of $x$ to be larger than that associated to $x$; for example, if $x$ has very small translation length and twist close to $\pi$, then $x^{2}$ will have a larger $(\log 3)$-cylinder.

The tubes that arise in our estimate are defined as follows. We consider the largest $(\log 3)$-cylinder associated to any power of $x$ and divide by the action of $x$. The radius, and hence the volume, of the resulting tube depends in quite an interesting way on the length and twist of $x$.

The body of this paper is divided into three sections. In $\S 1$ we establish notation and review some basic geometric facts. In $\S 2$ we prove, using the $\log 3$ Theorem of [2], that if $\beta_{1}(M) \geq 2$ and if $\lambda<\log 3$, then either $M$ contains a hyperbolic ball of radius $\lambda / 2$ or a tube associated with an element $x \in \pi_{1}(M)$ of translation length less than $\lambda$. To carry out the estimate we choose a good value for $\lambda$, namely, one such that the volume of the tube associated with any isometry of length less than $\lambda$ exceeds the lower bound, given by density estimates for sphere-packings, for the volume of a manifold containing a hyperbolic ball of radius $\lambda / 2$. The analysis underlying this choice is described in $\S 3$.

\section{Notation}

1.1. We will identify the group of orientation-preserving isometries of hyperbolic 3-space $\mathbf{H}^{3}$ with the group of Möbius transformations of the upper half-space model. A loxodromic isometry will be said to have complex length $\alpha$ if it is conjugate to the Möbius transformation $z \mapsto e^{\alpha} z$. If $x$ has complex length $\alpha$ then the real and imaginary parts of $\alpha$ will be called, respectively, the translation length and twist of $x$. We will say that an isometry of $\mathbf{H}^{3}$ is $\lambda$-short if it is loxodromic and has translation length less than $\lambda$. 
1.2. If $M$ is a hyperbolic 3-manifold then $M$ may be regarded as the quotient of $\mathbf{H}^{3}$ by a torsion-free discrete group $\Gamma$ of hyperbolic isometries. If $M$ is closed then $\Gamma$ is cocompact and each nontrivial element of $\Gamma$ is loxodromic. In this case the centralizer of a nontrivial element $x$ of $\Gamma$ is the maximal cyclic subgroup of $\Gamma$ containing $x$. The centralizer of $x$ can also be characterized as the subgroup of $\Gamma$ consisting of all elements which keep the axis of $x$ invariant.

1.3. Suppose that $x$ is a loxodromic isometry of $\mathbf{H}^{3}$ with complex length $\alpha=l+i \theta$. It will be useful to have a formula for the displacement of $x$, i.e., the distance from a point of $\mathbf{H}^{3}$ to its image under $x$ (see [3]). If $p$ is a point at a distance $R$ from the axis of $x$ then

$$
\cosh \operatorname{dist}(p, x(p))=\cosh l+\sinh ^{2}(R)(\cosh l-\cos \theta) .
$$

We will write

$$
Z_{\lambda}^{n}(x)=\left\{p \in \mathbf{H}^{3} \mid \operatorname{dist}\left(p, x^{k}(p)\right)<\lambda \text { for some } k \text { with } 1 \leq k \leq n\right\}
$$

and

$$
Z_{\lambda}(x)=\bigcup_{n \geq 1} Z_{\lambda}^{n}(x)=\left\{p \in \mathbf{H}^{3} \mid \operatorname{dist}\left(p, x^{k}(p)\right)<\lambda \text { for some } k \geq 1\right\} .
$$

If $x$ has complex length $l+i \theta$ then $Z_{\lambda}^{1}(x)$ is empty if $l \geq \lambda$ and otherwise is a circular cylinder about the axis of $x$ with radius $R$ satisfying

$$
\sinh ^{2}(R)=\frac{\cosh \lambda-\cosh l}{\cosh l-\cos \theta}
$$

The quotient $Z_{\lambda}^{1}(x) /\langle x\rangle$ is a tube which has volume

$$
\pi l \sinh ^{2}(R)=\pi l\left(\frac{\cosh \lambda-\cosh l}{\cosh l-\cos \theta}\right) .
$$

It follows that $Z_{\lambda}(x) /\langle x\rangle$ is a tube of volume

$$
\pi l \max _{n \geq 1}\left(\frac{\cosh \lambda-\cosh n l}{\cosh n l-\cos n \theta}\right) .
$$

The following observation, which is immediate from the definitions, will be used throughout.

If $x$ and $y$ are isometries of $\mathbf{H}^{3}$ then $Z_{\lambda}(x) \cap Z_{\lambda}(y) \neq \varnothing$ if and only if there exists $p \in \mathbf{H}^{3}$ and nonzero integers $m$ and $n$ with

$$
\max \left(\operatorname{dist}\left(p, x^{n}(p)\right), \operatorname{dist}\left(p, y^{m}(p)\right)\right)<\lambda .
$$

1.4. A finitely generated Kleinian group $\Gamma$ is said to be topologically tame if the quotient 3-manifold $\mathbf{H}^{3} / \Gamma$ is homeomorphic to the interior of a compact 3-manifold.

\section{HYPERBOLIC BALLS AND TUBES}

2.1. The following consequence of the $\log 3$ Theorem of [2] is the basis for the volume estimate in this paper. 
Proposition. Let $M=\mathbf{H}^{3} / \Gamma$ be a closed orientable hyperbolic 3-manifold. Let $\lambda<\log 3$ be given. Suppose that $x$ is a $\lambda$-short element of $\Gamma$ which is not a proper power. If every subgroup of $\Gamma$ generated by two conjugates of $x$ is of infinite index and topologically tame, then $M$ contains an open set isometric to $Z_{\log 3}(x) /\langle x\rangle$.

Proof. To prove that $M$ contains an open set isometric to $Z_{\log 3}(x) /\langle x\rangle$, it suffices to show that under every element of $\Gamma$ the cylinder $Z_{\log 3}(x)$ either is invariant or is mapped to a disjoint cylinder. The subgroup which keeps $Z_{\log 3}(x)$ invariant is exactly the centralizer of $x$, which is generated by $x$ since $x$ is not a proper power. Thus it suffices to show that if $y \in \Gamma$ does not commute with $x$ then $y$ maps $Z_{\log 3}(x)$ to a disjoint cylinder, i.e., that $Z_{\log 3}(x) \cap Z_{\log 3}\left(y x y^{-1}\right)=\varnothing$. By 1.3 we must show that if $y$ does not commute with $x$ then for any nonzero integers $m$ and $n$ and any $p \in \mathbf{H}^{3}$ we have $\max \left(\operatorname{dist}\left(p, x^{n}(p)\right), \operatorname{dist}\left(p, y x^{m} y^{-1}(p)\right)\right) \geq \log 3$.

The $\log 3$ Theorem of [2] states that if $\xi$ and $\eta$ are noncommuting hyperbolic isometries which generate a torsion-free discrete group that is topologically tame, is not co-compact, and contains no parabolics, then

$$
\max (\operatorname{dist}(p, \xi(p)), \operatorname{dist}(p, \eta(p))) \geq \log 3 \text { for any } p \in \mathbf{H}^{3} .
$$

Since $M$ is closed, $\Gamma$ contains no parabolics. A subgroup of infinite index in $\Gamma$ is necessarily non-co-compact. We wish to apply the $\log 3$ Theorem with $\xi=x^{n}$ and $\eta=y x^{m} y^{-1}$, which clearly generate a subgroup of infinite index since $x$ and $y$ do. Moreover, the group generated by $x^{n}$ and $y x^{m} y^{-1}$ is topologically tame since, by [1, Proposition 3.2], a finitely generated subgroup of an infinite-volume topologically tame Kleinian group is topologically tame.

Thus we need only show that if $y$ does not commute with $x$ and if $m$ and $n$ are nonzero integers, then $x^{n}$ and $y x^{m} y^{-1}$ do not commute. This follows from 1.2 since the axis of $y x^{m} y^{-1}$ is the image under $y$ of the axis of $x$.

2.2. Proposition. Let $M=\mathbf{H}^{3} / \Gamma$ be a closed orientable hyperbolic 3-manifold. Suppose that $x$ and $y$ are elements of $\Gamma$ which are contained in the kernel of a homomorphism from $\Gamma$ onto $\mathbf{Z}$. Then $\langle x, y\rangle$ is topologically tame and of infinite index in $\Gamma$.

Proof. The proof of this statement is the main step in the proof of [2, Proposition 10.2].

2.3. Proposition. Let $M=\mathbf{H}^{3} / \Gamma$ be a closed orientable hyperbolic 3-manifold with $\beta_{1}(M) \geq 2$. Let $\lambda<\log 3$ be given. Either $M$ contains a hyperbolic ball of radius $\lambda / 2$ or else $M$ contains an open set isometric to $Z_{\log 3}(x) /\langle x\rangle$, where $x$ is some $\lambda$-short element of $\Gamma$.

Proof. If every element of $\Gamma$ has translation length greater than $\lambda$, then the injectivity radius at every point of $M$ is greater than $\lambda / 2$. In particular $M$ contains a ball of radius $\lambda / 2$.

Otherwise, let $x \in \Gamma$ have translation length less than $\lambda$. We may assume that $x$ is not a proper power. Since $\beta_{1}(M) \geq 2$, any subgroup of $\Gamma$ which is generated by two conjugates of $x$ is contained in the kernel of a homomorphism onto $\mathbf{Z}$ and is thus topologically tame and of infinite index in $\Gamma$ by Proposition 2.2. By Proposition 2.1, $M$ contains an open set isometric to $Z_{\log 3}(x) /\langle x\rangle$. 


\section{The estimate}

3.1. The first step in obtaining a concrete lower bound for volume from Proposition 2.3 is to make an appropriate choice of a number $\lambda<\log 3$. We choose $\lambda=0.8$. Using density estimates for sphere-packings as in [7] (see also [2]), one computes that the volume of a hyperbolic 3-manifold which contains a hyperbolic ball of radius $\lambda / 2=0.4$ is at least 0.35 . The two lemmas which are proved in this section immediately imply the following.

Proposition. If $x$ is a loxodromic isometry of $\mathbf{H}^{3}$ with translation length less than 0.8 then the volume of $Z_{\log 3}(x) /\langle x\rangle$ is greater than 0.34 .

Taking $\lambda=0.8$ in Proposition 2.3 we thus obtain our volume estimate for hyperbolic manifolds with Betti number 2 .

Theorem. If $M$ is a closed orientable hyperbolic 3-manifold with $\beta_{1}(M) \geq 2$ then the volume of $M$ exceeds 0.34 .

3.2. When the translation length of our loxodromic isometry $x$ is very short we can use a lemma of Zagier's [8, p. 1045] to give a lower bound for the volume of $Z_{\log 3}(x) /\langle x\rangle$.

Lemma. If $x$ is a loxodromic isometry of $\mathbf{H}^{3}$ with translation length less than 0.065 then the volume of $Z_{\log 3}(x) /\langle x\rangle$ is at least 0.34 .

Proof. Let $x$ have complex length $l+i \theta$, where $0<l<0.065$. Zagier's Lemma states that if $\theta$ and $l$ are real numbers with $0<l<\pi \sqrt{3}$ then there exists an integer $n_{0} \geq 1$ such that

$$
\cosh n_{0} l-\cos n_{0} \theta \leq \cosh \sqrt{\frac{4 \pi l}{\sqrt{3}}}-1 \text {. }
$$

Note that this implies that

$$
\cosh n_{0} l \leq \cosh \sqrt{\frac{4 \pi l}{\sqrt{3}}}
$$

Now, substituting into (1.3.1) and using $\cosh (\log 3)=5 / 3$, we have

$$
\begin{aligned}
\operatorname{vol} Z_{\log 3}(x) /\langle x\rangle & =\pi l \max _{n>0}\left(\frac{5 / 3-\cosh n l}{\cosh n l-\cos n \theta}\right) \\
& \geq \pi l\left(\frac{5 / 3-\cosh n_{0} l}{\cosh n_{0} l-\cos n_{0} \theta}\right) \\
& \geq \pi l\left(\frac{5 / 3-\cosh \sqrt{4 \pi l / \sqrt{3}}}{\cosh \sqrt{4 \pi l / \sqrt{3}}-1}\right) \doteq \mu(l) .
\end{aligned}
$$

One checks that $\mu$ is decreasing on $[0,0.065]$ and that $\mu(0.065)=$ $0.3509826 \ldots$.

3.3. Our volume estimate is completed by the following lemma. The proof is simply a numerical computation, albeit one of sufficient complexity to require the use of a computer. 
Lemma. If $x$ is a loxodromic isometry of $\mathbf{H}^{3}$ with translation length in the interval $[0.065,0.8]$ then the volume of $Z_{\log 3}(x) /\langle x\rangle$ is at least 0.34 .

Proof. Let $x$ have complex length $l+i \theta$. Recall that

$$
\operatorname{vol} Z_{\log 3}(x) /\langle x\rangle=\pi l \max _{n \geq 1}\left(\frac{5 / 3-\cosh n l}{\cosh n l-\cos n \theta}\right) .
$$

Set

$$
f_{n}(l, \theta)=\pi l\left(\frac{5 / 3-\cosh n l}{\cosh n l-\cos n \theta}\right) .
$$

We must show that 0.34 is a lower bound for $\max _{n \geq 1} f_{n}(l, \theta)$ for $(l, \theta)$ in the rectangle $R=[0.065,0.8] \times[0, \pi]$. Clearly it suffices to show that 0.34 is a lower bound for $g(l, \theta)=\max _{1 \leq n \leq 7} f_{n}(l, \theta)$. Note that $g$ is a maximum of a finite number of differentiable functions; in particular, it is continuous.

We make some observations about the functions $f_{n}$ which can be verified by elementary calculus:

(i) $f_{n}$ has no local extrema in the interior of $R$;

(ii) $\partial f_{n} / \partial l=0$ implies $\partial^{2} f_{n} / \partial l^{2}<0$;

(iii) For fixed $l$, the local minima of $f_{n}(l, \theta)$ are all equal to the global minimum and occur where $n \theta=m \pi$ for an odd integer $m$; in this case we have $f_{n}(l, \theta)=f_{n}(l, \pi / n)$.

Suppose that $R_{0}=[a, b] \times[c, d]$ is a rectangle contained in $R$. It follows from the three facts above that the minimum value of $f_{n}$ on $R_{0}$ is attained at one of the four corners of $R_{0}$ unless there exists an odd integer $m$ such that $(m / n) \pi \in[c, d]$. In the latter case the minimum is either $f_{n}(a, \pi / n)$ or $f_{n}(b, \pi / n)$. The point here is that the minimum of $f_{n}$ on $R_{0}$ can be computed by evaluating $f_{n}$ at either two or four points, the coordinates of which can be computed in terms of $a, b, c, d$.

Note that $\max _{1 \leq n \leq 7} \min _{R_{0}} f_{n}$, truncated to a prescribed number of digits, gives a lower bound for $g$ on the rectangle $R_{0}$ and can be computed by making no more than 28 function evaluations. Thus the following algorithm will produce an explicit lower bound $\kappa$ for $g$ on $R$ : subdivide $R$ into product rectangles $R_{i j}, 1 \leq i \leq N, 1 \leq j \leq M$; compute a truncation $\kappa_{i j}$ of $\max _{1 \leq n \leq 7} \min _{R_{i j}} f_{n}$; and compute $\kappa=\min _{i, j} \kappa_{i j}$.

For programming convenience we used a partition of the rectangle $R$ into $320 \times 200$ equal-sized rectangles when carrying out the above algorithm by computer. (A somewhat coarser partition could be used, although $50 \times 20$ is not fine enough.) The computation produced a lower bound $\kappa=0.343$, which establishes the Proposition.

3.4. The computer study used to prove 3.3 gives considerable qualitative information about the behavior of the volume of $Z_{\log 3}(x) /\langle x\rangle$ as a function of complex length. This is illustrated in Figures 1 and 2.

One conjecture suggested by this study is that the volume of $Z_{\log 3}(x) /\langle x\rangle$ does not tend uniformly to infinity as the translation length approaches 0 . If $x$ has complex length $l+i \theta$ where $\theta$ is a fixed rational multiple of $\pi$ then the volume tends to infinity as $l$ tends to 0 . However it appears from the computation that there is a sequence of isometries $x_{i}$ with translation lengths tending to 0 so that $\operatorname{vol} Z_{\log 3}\left(x_{i}\right) /\left\langle x_{i}\right\rangle$ approaches $1 / \sqrt{3}$, which is the limiting value as $l \rightarrow 0$ of the lower bound $\mu(l)$ based on Zagier's lemma. 


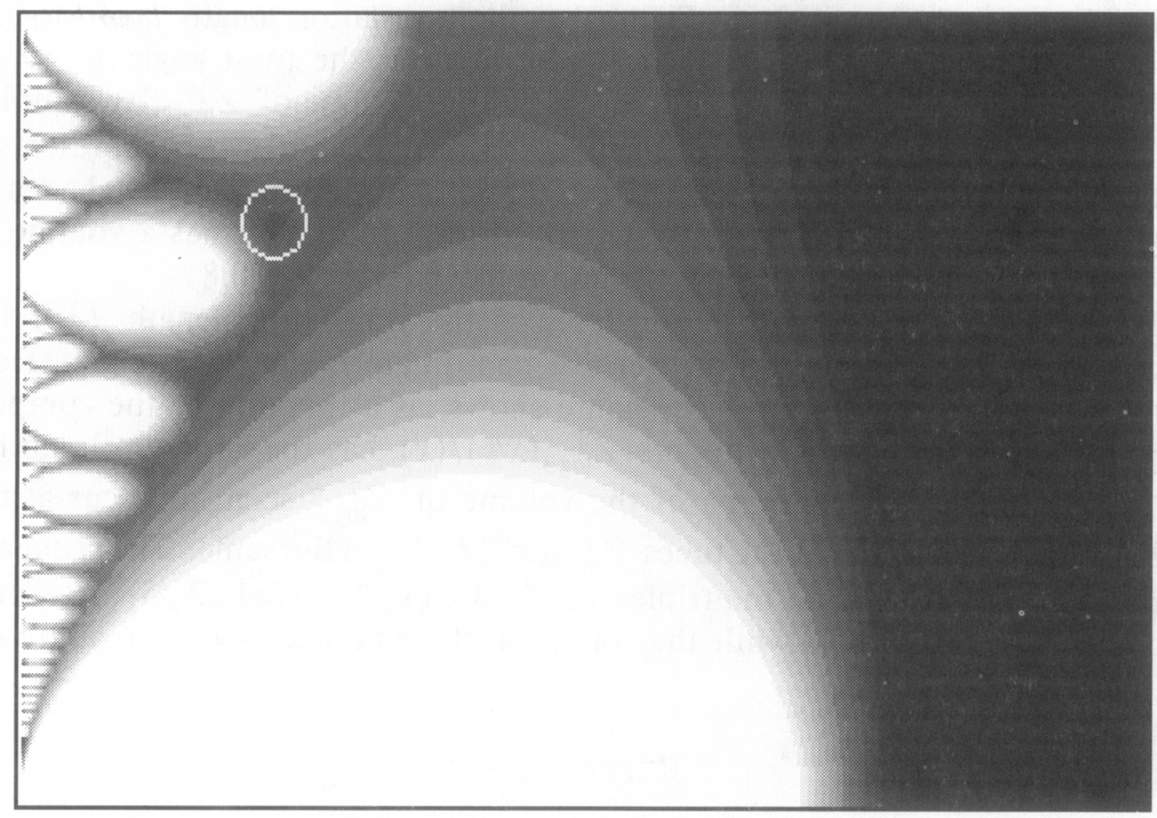

FIGURE 1. The volume of $Z_{\log 3}(x) /\langle x\rangle$ as a function of complex length.

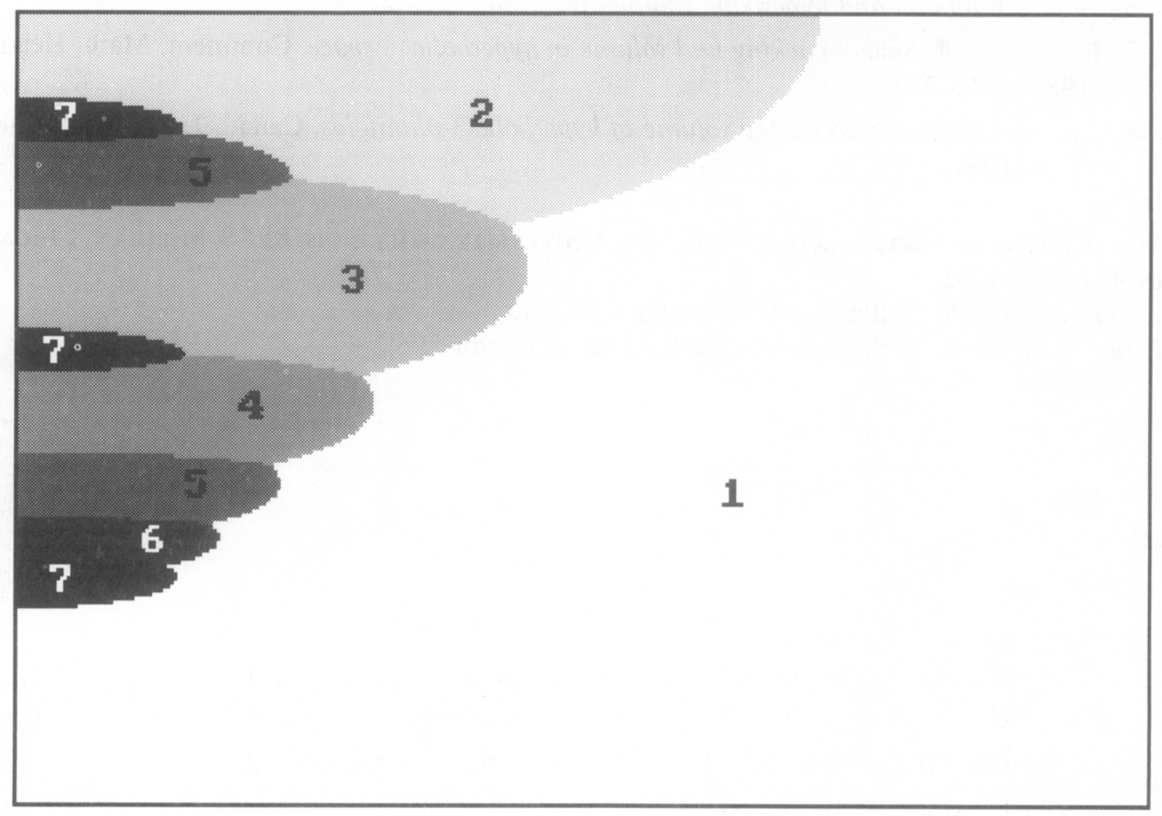

FIGURE 2. Power $m \leq 7$ yielding the largest volume tube. 
The horizontal coordinate in Figure 1 is the translation length $l$ of the loxodromic isometry $x$, and the vertical coordinate is the twist angle $\theta$. Here $0<l<1.4$ and $0<\theta<\pi$. The figure shows the volume of $Z_{\log 3}^{30}(x)$ sampled on a $320 \times 200$ grid. Black represents a volume of 0 and white represents a volume greater than 1 . Volumes between 0 and 1 are represented by shades of gray, with smaller values darker. The circled local minimum has a volume of $0.34588 \ldots$, making it the global minimum for $0.065<l<0.8$.

The horizontal coordinate in Figure 2 is the translation length $l$ of the loxodromic isometry $x$, and the vertical coordinate is the twist angle. Here $0<l<0.7$ and $0<\theta<\pi$. The figure shows, as a function of the complex length of $x$, which of the cylinders $Z_{\log 3}^{1}\left(x^{m}\right) /\langle x\rangle$ has the largest volume for $1 \leq m \leq 7$. The local minima of the volume of $Z_{\log 3}^{7}$ seem to occur at the eight points where three of the tubes $Z_{\log 3}^{1}\left(x^{m}\right) /\langle x\rangle$ are the same. The sequence of points corresponding to the triples $(1,2,3),(1,2,4),(1,2,5), \ldots$ have $l$-coordinates tending to 0 while the volume at these points appears to approach $1 / \sqrt{3}$.

\section{REFERENCES}

1. R. Canary, Ends of hyperbolic 3-manifolds, preprint.

2. M. Culler and P. B. Shalen, Paradoxical decompositions, 2-generator Kleinian groups, and volumes of hyperbolic 3-manifolds, J. Amer. Math. Soc. 5 (1992), 231-288.

3. __ Hyperbolic volume and mod p homology, preprint.

4. _ Volumes of hyperbolic Haken manifolds. I, preprint.

5. F. Gehring and G. Martin, Inequalities for Möbius transformations and discrete groups, preprint.

6. __ 6-torsion and hyperbolic volume, preprint.

7. R. Meyerhoff, Sphere-packing and volume in hyperbolic 3-space, Comment. Math. Helv. 61 (1986), 271-278.

8. $\ldots$ A lower bound for the volume of hyperbolic 3-manifolds, Canad. J. Math. 34 (1987), 1038-1056.

Department of Mathematics (M/C 249), University of Illinois, 851 S. Morgan, Chicago, ILLINOIS 60607-7045

E-mail address, M. Culler: culler@math.uic.edu

E-mail address, P. B. Shalen: u10123Quicvm.uic.edu 